\title{
Diverse muscle architecture adaptations in a rabbit tibial lengthening model
}

\author{
Mitsuhiko Takahashi \\ Natsuo Yasui \\ Tetsuya Enishi \\ Nori Sato \\ Takatoshi Mizobuchi \\ Yukako Homma \\ Koichi Sairyo
}

The Institute of Health BioSciences, the University of Tokushima, Japan

Corresponding author:

Mitsuhiko Takahashi

3-18-15 Kuramoto,

770-8503 Tokushima, Japan

E-mail:w-tk@umin.ac.jp

\section{Summary}

Background: during limb lengthening, muscles are thought to increase the number of sarcomeres. However, this adaptation may differ among muscles with diverse architecture. Purpose: this study wish to clarify the differences in muscle adaptation in a rabbit model of tibial lengthening. Methods: twelve rabbits underwent tibial lengthening $(0.7 \mathrm{~mm} /$ day for 4 weeks), with the contralateral limb serving as a control, and were euthanized after either the lengthening or the consolidation period. Six muscles around the tibia were investigated in terms of muscle belly length, muscle weight, sarcomere length and serial sarcomere number. Results: muscle belly length increased in all the lengthened muscles. No increases in muscle mass were noted. Sarcomere length increased in the ankle plantar-flexors and was kept longer than the optimal sarcomere length after the consolidation period. Nevertheless, significant increases in sarcomere number were observed in two ankle plantar-flexors. Conclusion: this study demonstrated that muscle belly length largely adapted to the lengthening. The increase in sarcomere number did not match the increase in muscle belly length. We estimated that elongation of the intramuscular aponeuroses is another mechanism of the adaptation in addition to the increase in sarcomere number.

KEY WORDS: aponeurosis, limb lengthening, muscle adaptation, muscle architecture, sarcomere number.

\section{Introduction}

Limb lengthening, that is, experimental distraction osteogenesis, has been widely used to treat various orthopedic diseases, such as congenital limb deficiency and acquired limb shortening. In this treatment, tension stress induces regeneration of all limb tissues including bone, muscle, vessels and skin $^{1,2}$. Despite the benefits of this treatment, several complications during limb lengthening have been reported ${ }^{3}$, in particular, muscle stiffness and joint contracture are major muscle-related complications. Equinus deformity is a well-known complication of tibial lengthening. Such complications are probably due to failure of the skeletal muscles to adapt to the substantial increase in muscle length.

Muscle contraction is generated by sarcomeres, the minimum functional units of muscle. Two major myofilaments, actin and myosin filaments, have consistent lengths in striated muscle. Since the contraction force in a sarcomere is generated at the overlap of actin and myosin filaments, sarcomere length is an important determinant of muscle force generation ${ }^{4}$. Human striated muscle can generate maximum active tension at sarcomere lengths of 2.6-2.8 $\mu \mathrm{m}$, which is considered an optimal sarcomere length ${ }^{5,6}$. On the other hand, sarcomeres elongated to the end of descending limb of the sarcomere length-tension relationship $(4.3 \mu \mathrm{m})$ can no longer generate active tension. Under this condition, muscle passive tension is undesirably increased, resulting in joint stiffness and contracture.

During limb lengthening treatment, sarcomere length is unavoidably increased by its treatment nature, leading to the loss of muscle contraction forces and an increase in muscle passive tension. To regain functional muscle contraction, muscles are thought to increase the number of sarcomeres in each muscle fiber by adding new sarcomeres in series to reduce sarcomere length ${ }^{7,8}$. This increase in sarcomere number is regarded as muscle adaptation to stretch. It is well known, however, that skeletal muscles have architectural diversity, specifically in fiber length and physiological cross-sectional area. In the lower leg, the tibialis anterior muscle has long fibers, while the soleus consists of many short fibers ${ }^{9}$. Muscle adaptation through increasing number of sarcomeres in series may differ among muscles with diverse architecture. Even in single-segment lengthening, where all muscles around the lengthening segment are lengthened in the same manner and to the same extent, muscle adaptation 
may be different. Against this background, this study sought to clarify the differences in muscle adaptation to a single-level stretch in a rabbit model of tibial lengthening, a model for which all muscle architectures have been previously described in literature ${ }^{10}$.

\section{Methods}

Experiments were performed in accordance with the guidelines of our Institutional Animal Care and Use Committee, and international ethical standard ${ }^{11}$. Twelve male Japanese White rabbits (mean body mass $\pm S D, 2.33 \pm 0.16 \mathrm{~kg}$ ) underwent tibial lengthening, with the contralateral limb serving as a control. A custom-made unilateral external fixator was fixed to the tibia with four half-pins, followed by mid-diaphyseal osteotomy between the second and third pins using a manual saw. The periosteum was sutured to cover the osteotomy site. After a latency period of 1 week, lengthening was started at a rate of $0.35 \mathrm{~mm}$ every $12 \mathrm{~h}$ ( $0.7 \mathrm{~mm} /$ day) and was stopped 5 weeks after the operation (total tibial lengthening, $19.6 \mathrm{~mm}$ in 4 weeks). Half of the animals $(n=6)$ were euthanized at the end of the lengthening, at which the lengthening was confirmed on radiographs (Group I). For the remaining animals $(n=6)$, a 4 -week consolidation period was instituted before being euthanized (Group II). The bilateral hindlimbs were then harvested for analysis in both groups. Radiographs were taken every 2 weeks after starting the lengthening protocol.

Hindlimbs were immersed for 5 days in $10 \%$ buffered formalin with the ankles and knees fixed at right angles. Six full-length muscles around the tibiae - the tibialis anterior (TA), extensor digitorum longus (EDL), lateral head of the gastrocnemius (GCL), soleus (Sol), flexor hallucis longus (FHL), and peroneus brevis (PB) - were detached from the tibiae with special care to include the originating tendinous tissue and the distal tendon. All muscles originated at the proximal to the lengthening site and inserted into distal to the ankle joint. All except TA are pinnate muscles, and all except Sol consist of mainly fast-twitch fibers ${ }^{10}$. Muscle belly length, defined as the length between the origin of the most proximal muscle fibers and the insertion of the most distal muscle fibers, and muscle wet weight (mass) were measured directly. Using a dissecting microscope, three full-length muscle fiber bundles were teased from the proximal and distal halves of each muscle (6 fiber bundles per muscle). The length of each teased muscle fiber bundle was measured directly using a digital caliper under the dissecting microscope. After mounting each fiber bundle on a glass slide, sarcomere length was measured at five points across the fiber bundle under a polarized light microscope (Eclipse TE2000-U, Nikon Corp., Tokyo, Japan) using a digital measuring system (Digital Sight DS-L2, Nikon Corp.). To obtain the mean sarcomere length at each point, 30 serial sarcomeres were measured and averaged (Fig. 1). The number of sarcomeres in series was calculated by dividing the fiber bundle length by the average sarcomere length measured at the five

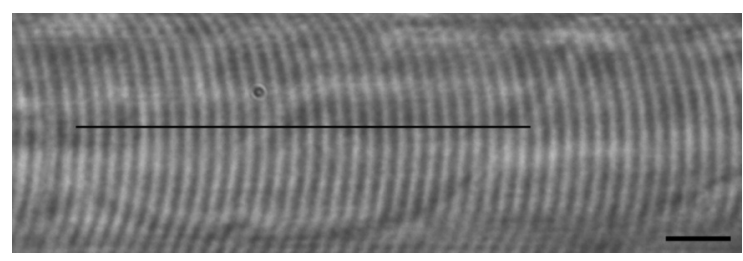

Figure 1. High-power field view of a teased muscle fiber bundle under a polarized microscope. Dark and light strata correspond to the A-band and I-band of the sarcomere, respectively. The line traversing the middle spans 30 sarcomeres, and the scale bar indicates $10 \mu \mathrm{m}$.

points. The mean fiber bundle length and sarcomere number were averaged from six measurements per muscle. The paired $t$-test was used to determine significant differences between the lengthened and contralateral control sides, and analysis of covariance was used for comparisons among six investigated muscles and between Group I and Group II at two time points, with the values of the contralateral side serving as covariates. If there was interactions between independent variables and covariates, analysis of variance was used for the comparisons. Data are presented as the means $\pm S D$, and $p<0.05$ was considered statistically significant.

\section{Results}

Radiography revealed uneventful bone formation in the lengthened site in all animals, and bony union of the lengthened gap after the 4-week consolidation period in Group II. Serial radiographs revealed that length of the contralateral tibiae increased in the form of natural bone growth by $8.5 \pm 1.8 \mathrm{~mm}$ and $14.8 \pm$ $2.5 \mathrm{~mm}$ during the 5-week course in Group I and during the 9-week course in Group II, respectively. Similarly, that of the lengthened tibiae increased in addition to the applied lengthening by $8.2 \pm 2.0 \mathrm{~mm}$ in Group I and $15.4 \pm 3.3 \mathrm{~mm}$ in Group II.

Muscle belly length increased significantly in all the lengthened muscles (range, 12.1-21.3 mm, 17.5$33.9 \%$ ) compared with the corresponding contralateral control muscles at the end of the lengthening period in Group I (Fig. 2). The increases in muscle belly length were maintained after the consolidation period in Group II (range 14.6-20.0 mm, 20.3-33.0\%). No significant differences were detected between Groups I and II or among the 6 muscles. Though the increase in muscle belly length did not completely match the applied tibial lengthening in this experiment; this outcome is not unreasonable since the elongation of each external tendon was involved in the adaptation to lengthening. In contrast to the increases in muscle belly length, no increases in muscle mass were noted in any lengthened muscles in either group compared with the corresponding contralateral muscles (Fig. 3). Furthermore, no significant differences in muscle mass were detected between Groups I and II or among the 6 muscles. These results indicate that 


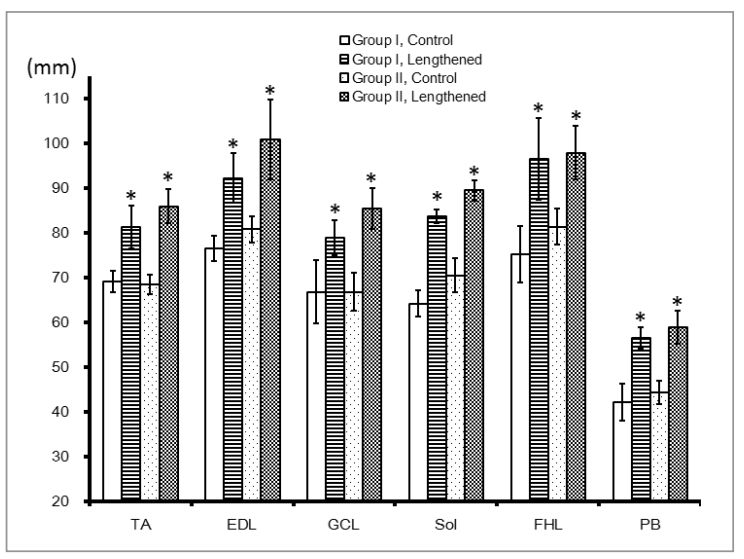

Figure 2. Muscle belly length of the 6 muscles investigated in this study. Each muscle is represented by 4 columns showing (from left to right) the data of the contralateral control and lengthened muscles at the end of the lengthening (Group I) and those of the contralateral control and lengthened muscles at the end of the consolidation period (Group II). Error bars indicate standard deviation. *Significant difference between lengthened and contralateral control sides (paired t-test, $\mathrm{p}<0.05$ ). \#Significant difference between the 6 muscles (analysis of covariance or analysis of variance, $p<0.05)$.

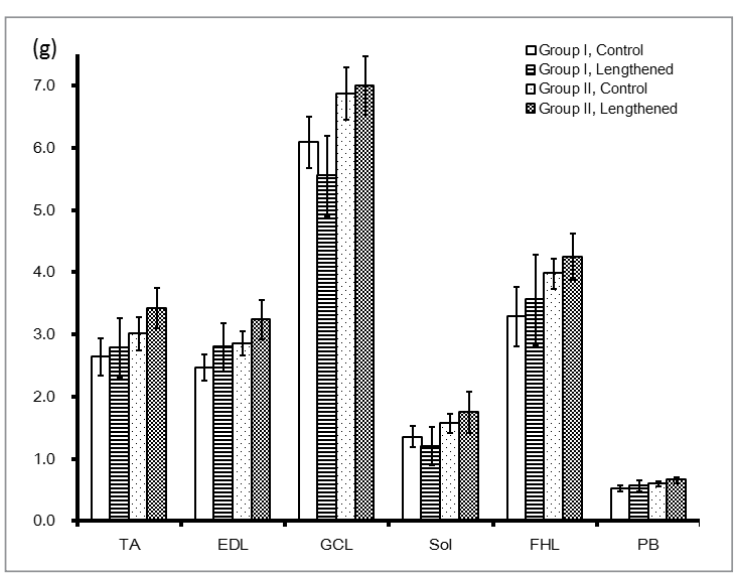

Figure 3. Mass of the 6 muscles investigated in this study. Descriptions are the same as Fig. 2.

limb lengthening increased muscle length but reduced cross-sectional area; in other words, lengthening resulted in muscle atrophy.

Sarcomere length in Group I increased significantly in the ankle plantar-flexors (GCL, Sol, and FHL) of the lengthened side $(2.53-2.61 \mu \mathrm{m})$ compared with the contralateral control side (2.08-2.27 $\mu \mathrm{m})$ (Fig. 4). Sarcomere length in TA, one of the ankle dorsiflexors, in Group I decreased compared with the contralateral side. Among the 6 investigated muscles, pairs in which the sarcomere length in the lengthened side was significantly different from that in the contralateral control side were TA-GCL, TA-FHL, EDL-GCL, and GCL-PB. Optimal sarcomere length in rabbit skeletal muscles has been estimated at 2.3-2.4 $\mu^{12}$. In the present study, the sarcomere length in the ankle plantar-flexors of the contralateral control side was less than the optimal sarcomere length, implying that these muscles are naturally adjusted to generate maximal active tension in ankle dorsiflexion, that is, in the stretched muscle position. On the lengthened side, however, sarcomere length in the ankle plantar-flexors was longer than the optimal sarcomere length, indicating that these plantarflexors would generate maximal tension during ankle plantar-flexion. This might present difficulties in ambulatory and jumping movements in normal rabbits. Sarcomere length in the plantar-flexors did not change between Group I and Group II, even though a 4-week consolidation period was instituted, which was considered a sufficient period for muscle adaptation. Sarcomere length in the lengthened EDL and PB decreased significantly during this 4-week period.

The number of sarcomeres in the contralateral control TA was about twice that of the other muscles, which seems to be a characteristic feature of parallel muscle. Significant increases in sarcomere number were observed in Sol $(52.1 \%)$ and FHL $(31.0 \%)$ of the lengthened side in Group I compared with the contralateral muscles (Fig. 5). The lengthened muscles in

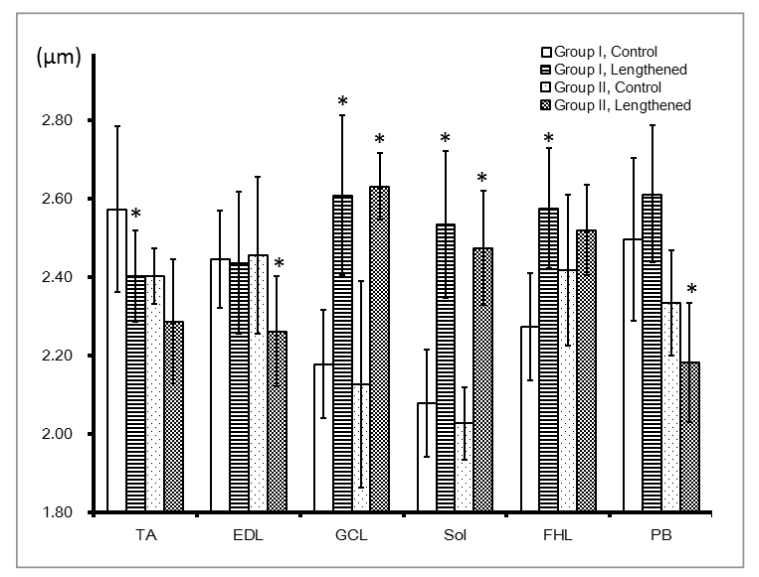

Figure 4. Sarcomere length of the 6 muscles investigated in this study. Descriptions are the same as Fig. 2.

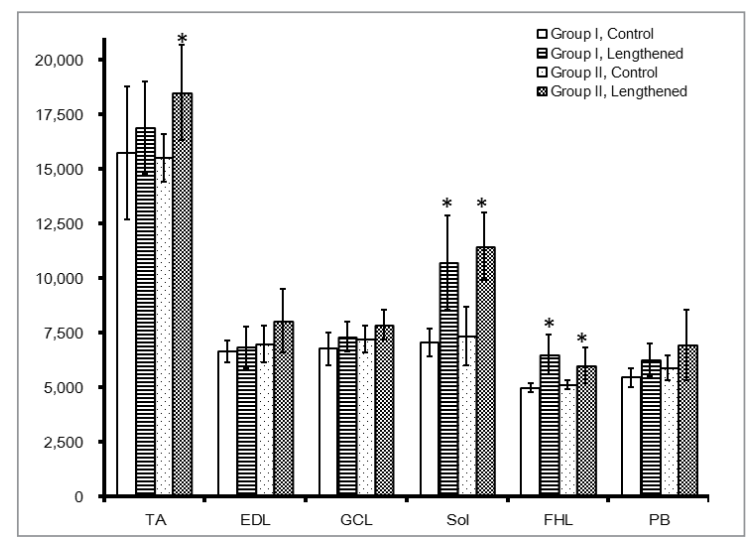

Figure 5. Number of sarcomeres in series of the 6 muscles investigated in this study. Descriptions are the same as Fig. 2. 
Group II had significantly more sarcomeres than the corresponding contralateral muscles in TA, Sol and FHL. Among the 6 muscles investigated, pairs in which the serial sarcomere number in the lengthened side was significantly different from that in the contralateral control side were TA-all other muscles and Sol-all other muscles.

\section{Discussion}

This study demonstrated that under a normal rate of lengthening $(0.7 \mathrm{~mm} /$ day $)$, all muscle belly lengths largely adapted to the applied lengthening. However, the increase in muscle belly length did not imply proportional increases in mass and number of sarcomeres. A rapid increase in number of sarcomeres $(\sim 3,700)$ was observed in Sol during the lengthening period, corresponding to an increase in muscle fiber length of approximately $10 \mathrm{~mm}$ when the sarcomere length was $\geq 2.5 \mu \mathrm{m}$; this increase was merely half the distance of the applied lengthening. These results suggest that muscle fibers are mostly elastically stretched while muscles becoming thinner by decreasing the fiber pennation angle, although other mechanisms might be involved in elongating the muscle belly length.

We considered elongation of the intramuscular aponeuroses as another mechanism. As all skeletal muscles have aponeuroses in the muscle belly, to which muscle fibers attach, muscles contain many muscle fibers within a compact space, which enables large contraction forces to be generated ${ }^{13}$. Previous studies have shown that the aponeurosis had a larger compliance compared with tendon ${ }^{14,15}$. The present tibial lengthening model used immature animals. A study with an experimental model of acute musculature stretch which closely investigated tendon adaptation showed that in young animals both tendon and muscle were elongated, while in adult animals only muscle contributed to the adaptation to the stretch ${ }^{16}$. In the present study, tendon measurement was not performed, which is one of the limitations of this study. However, measurement of tendinous elements in the same rabbit tibial lengthening model is currently underway. It will also be necessary to clarify whether this adaptation is due to aponeurosis compliance, aponeurosis remodeling, or regeneration of aponeurosis elements.

It is well known that muscles have a high adaptability to mechanical demands and that the number of sarcomeres in series could be easily increased to regain an optimal sarcomere length ${ }^{17,18}$. This sarcomerogenesis was recently confirmed by a mathematical model based on experimental data ${ }^{19}$. However, in the present study, a significant increase in number of sarcomeres was found in only Sol and FHL during the lengthening period. Sol, which showed the highest increase in number of sarcomeres, contains primarily type I (slow-twitch oxidative) fibers and has been shown to have larger adaptability to the applied stretch than any other muscles ${ }^{20}$. There are no other slow-twitch dominant muscles in the lower hindlimb of the rabbit ${ }^{10}$. The increase in number of sarcomeres was less conspicuous in other muscles even after the 4-week consolidation period, the reason for which should be clarified in future studies. It is not known whether this higher adaptability of Sol to lengthening is specific to Sol or common to the other slow-twitch oxidative muscles. Type I muscle fiber typically presents a two- to threefold higher mitochondrial density ${ }^{21}$. Recently it has been recognized that mitochondrial behavior regulates not only muscle function but muscle growth and myoblast differentiation ${ }^{21,22}$. The significant increase in number of sarcomere in Sol might be explained by mitochondrial specialization.

No additional increase in number of sarcomeres was observed during the consolidation period, even though the sarcomere length of Sol was higher than that of the control side at the end of lengthening. Sarcomere length in other ankle plantar-flexors of the lengthened side was increased compared with that in the corresponding contralateral muscles. These results may be related to equinus contracture, which is sometimes encountered in clinical limb lengthening. Recently, a goat hindlimb lengthening model showed that the ankle plantar-flexors had less capacity for adaptation to lengthening than the dorsiflexors, and one of the reasons for this difference was the larger muscle volume in the plantar-flexors compared with the dorsiflexors ${ }^{23}$. Clarifying the detailed mechanism of poor adaptability of the plantar-flexors should be also done in the future study and it will be important target for improving limb function after limb lengthening.

\section{References}

1. Ilizarov GA. The tension-stress effect on the genesis and growth of tissues. Part I. The influence of stability of fixation and soft-tissue preservation. Clin Orthop Relat Res. 1989; 238:249-281.

2. Ilizarov GA. The tension-stress effect on the genesis and growth of tissues: Part II. The influence of the rate and frequency of distraction. Clin Orthop Relat Res. 1989;239:263-285.

3. Paley D. Problems, obstacles, and complications of limb lengthening by the llizarov technique. Clin Orthop Relat Res. 1990;250:81-104.

4. Huxley AF, Simmons RM. Proposed mechanism of force generation in striated muscle. Nature. 1971;233(5321):533-538.

5. Herzog W, ter Keurs HE. Force-length relation of in-vivo human rectus femoris muscles. Pflugers Arch. 1988;411(6):642647.

6. Walker SM, Schrodt GR. I segment lengths and thin filament periods in skeletal muscle fibers of the Rhesus monkey and the human. Anat Rec. 1974;178(1):63-81.

7. Friden J, Ponten E, Lieber RL. Effect of muscle tension during tendon transfer on sarcomerogenesis in a rabbit model. $J$ Hand Surg [Am]. 2000;25(1):138-143.

8. Takahashi M, Ward SR, Marchuk LL, Frank CB, Lieber RL. Asynchronous muscle and tendon adaptation after surgical tensioning procedures. J Bone Joint Surg Am. 2010;92(3):664674.

9. Ward SR, Eng CM, Smallwood LH, Lieber RL. Are current measurements of lower extremity muscle architecture accurate? Clin Orthop Relat Res. 2009;467(4):1074-1082. 
10. Lieber RL, Blevins FT. Skeletal muscle architecture of the rabbit hindlimb: functional implications of muscle design. J Morphol. 1989;199(1):93-101.

11. Padulo J, Oliva F, Frizziero A, Maffulli N. Muscles, Ligaments and Tendons Journal. Basic principles and recommendations in clinical and field science research. MLTJ. 2013;4:250-252.

12. Sosa H, Popp D, Ouyang G, Huxley HE. Ultrastructure of skeletal muscle fibers studied by a plunge quick freezing method: myofilament lengths. Biophys J. 1994;67(1):283-292

13. Rehorn MR, Blemker SS. The effects of aponeurosis geometry on strain injury susceptibility explored with a 3D muscle model. J Biomech. 2010;43(13):2574-2581.

14. Lieber RL, Leonard ME, Brown CG, Trestik CL. Frog semitendinosis tendon load-strain and stress-strain properties during passive loading. Am J Physiol. 1991;261(1):C86-C92.

15. Zuurbier CJ, Everard AJ, van der Wees $P$, Huijing PA. Lengthforce characteristics of the aponeurosis in the passive and active muscle condition and in the isolated condition. J Biomech. 1994;27(4):445-453.

16. Tardieu C, Tabary JC, Tabary C, Huet de la Tour E. Comparison of the sarcomere number adaptation in young and adult animals. Influence of tendon adaptation. J Physiol (Paris). 1977;73(8):1045-1055.
17. Tabary JC, Tabary C, Tardieu C, Tardieu G, Goldspink G. Physiological and structural changes in the cat's soleus muscle due to immobilization at different lengths by plaster casts. $J$ Physiol. 1972;224(1):231-244.

18. Williams PE, Goldspink $G$. The effect of immobilization on the longitudinal growth of striated muscle fibres. J Anat. 1973; 116(Pt 1):45-55.

19. Zollner AM, Abilez OJ, Bol M, Kuhl E. Stretching skeletal muscle: chronic muscle lengthening through sarcomerogenesis. PLoS One. 2012;7(10):e45661.

20. Spector SA, Simard CP, Fournier M, Sternlicht E, Edgerton VR. Architectural alterations of rat hind-limb skeletal muscles immobilized at different lengths. Exp Neurol. 1982;76(1):94-110.

21. Picard M, Hepple RT, Burelle Y. Mitochondrial functional specialization in glycolytic and oxidative muscle fibers: tailoring the organelle for optimal function. Am J Physiol Cell Physiol. 2012;302(4):C629-C641.

22. Barbieri $E$, Sestili $P$, Vallorani $L$, et al. Mitohormesis in muscle cells: a morphological, molecular, and proteomic approach. MLTJ. 2013;3(4):254-266.

23. Makarov M, Birch J, Samchukov M. The role of variable muscle adaptation to limb lengthening in the development of joint contractures: an experimental study in the goat. J Pediatr Orthop. 2009;29(2):175-181. 